from controls in schizophrenic-like or dissociative symptoms.

Discussion: The dose-dependent hypopriming effect at the long SOA induced by acute ketamine was indicative of controlled processing impairments. In ketamine users, there was also an indication of controlled processing impairments and a suggestion that longterm ketamine abuse results in damage to the semantic store.

\section{Behavioural and fMRI evidence of semantic category deficits in schizophrenia}

\section{S Rossell, I Labuschagne}

Mental Health Research Institute of Victoria, Melbourne, Australia

Background: Abnormalities in semantic processing are commonly proposed to be central to cognitive abnormalities and thought disturbances in schizophrenia. Deficits have been reported on a range of tasks including a categorization task. The current study investigated the underlying neural substrates involved during categorization.

Method: A revised version of Chen et al.'s (1994) categorization task was used. The task consisted of 18 categories with five different exemplar words (ie high frequency, low frequency, borderline, related but outside category and unrelated) selected for each category. Subjects were asked to say whether exemplars were or were not part of the category. Data for each exemplar type were examined; this included behavioural accuracy and an event-related analysis of the functional magnetic resonance imaging data using SPM2.

Results: Behaviourally, patients with schizophrenia had difficulty categorizing related words, while the controls had most difficulty with borderline examples. Performance in the controls was related to activity in the left inferior frontal, left inferior occipital/posterior temporal, bilateral precunues and the cerebellum; areas typically reported during semantic processing. Even when behavioural performance on some of the category types was no different to control performance, the patients with schizophrenia did not show any activation of this network.

Conclusions: The imaging data showed impairments in the distributed frontal temporal network that is engaged in the representation and processing of meaning of words, text and discourse. It is these abnormalities that may underlie schizophrenic thought disturbance.

\section{Jumping to conclusions in delusions: fact or fallacy?}

\author{
S Rossell, A O'Regan, N Joshua
}

Mental Health Research Institute, Melbourne, Australia

Background: A jumping-to-conclusions (JTC) bias refers to the gathering of minimal data when making probabilistic judgments and has been associated with delusion formation. Approximately $50 \%$ of patients who experience delusions have previously been shown to have a JTC bias. However, the literature is fraught with methodological differences. This study sought to address one of these differences by examining state and trait delusions using large groups of patients with psychosis.

Methods: Three matched groups (patients with bipolar disorder, patients with schizophrenia and healthy controls) completed the standard beads probabilistic reasoning task and two emotional variants, which examined reasoning using personality and health traits. For each of the three tasks, two probability ratios were used, 85:15 and 60:40. JTC was defined, using the Institute of Psychiatry, London, criteria, as requiring two or less draws. Patients were divided into those with state and trait delusions.

Results: On the beads $85: 15$ task, only $4 \%$ of patients with schizophrenia and $0 \%$ of patients with bipolar disorder showed a JTC bias. The emotional variants produced a similar degree of JTC bias. The 60:40 ratio resulted in an even smaller proportion of JTC. There were no state-trait differences.

Discussion: Two groups of Australian patients with psychosis (schizophrenia and bipolar disorder) did not show a JTC bias. The authors discuss possible explanations for the discrepancy of these findings with the literature. These include the following: are Australians more indecisive or cautious, how state and trait delusions influence performance, and methodological problems with the task itself.

\section{Building and evaluating effective mental health networks in rural communities}

\author{
G Sartore', B Kelly', L Fragar², J Fuller³ , H Stain'1, \\ A Tonna', G Pollard' \\ 'Centre for Rural and Remote Mental Health, University of Newcastle; \\ ${ }^{2}$ Australian Centre for Agricultural Health and Safety, The University of Sydney; \\ and ${ }^{3}$ Northern Rivers University Department of Rural Health, \\ The University of Sydney, Sydney, Australia
}

Background: Rural communities suffer significant disadvantage in accessing mental health services. Conversely, these communities may have greater capacity 
to develop and maintain networks of nontraditional service providers to compensate for this lack of access; however, many agencies are unaware of each others' roles in maintaining the health of the community. A novel method of building and evaluating mental health service networks was trailed in rural New South Wales. Methods: Workshops providing education and support for agencies involved in providing services to rural communities were held in 12 locations. Participants' knowledge of mental health issues and the role of other support agencies, and their confidence in using this knowledge, were evaluated by pre- and postworkshop surveys. A new method of evaluating existing network strength and efficacy, using structured key informant interviews and network analysis methods, was trailed in several locations. Local service networks were reevaluated following the workshops to determine the extent, direction and determinants of network change. Results: About 96 workshop participants completed pre- and postworkshop surveys. Significant improvements in knowledge, confidence and social distance were found. Local referral networks were successfully mapped and changes in local communities following the workshops were evaluated.

Conclusions: Existing networks of service providers in rural communities have substantial capacity to act in nontraditional ways to improve the mental health of those communities. A relatively simple intervention, combined with ongoing support from local health agencies, can increase this capacity and the strength and efficiency of networks.

\section{A taxometric exploration of alcohol and cannabis problems in a community sample}

\section{T Slade', M Teesson ${ }^{2}$}

'University of New South Wales; and ${ }^{2}$ National Drug and Alcohol Research Centre, Sydney, Australia

Background: There is growing evidence that problems associated with substance use disorders (eg dependence and abuse) are most appropriately conceptualized using one or more continuous dimensions rather than as categorical 'yes/no' entities. Taxometric analysis is a statistical technique specifically designed to test this assertion.

Method: DSM-IV symptoms associated with the use of alcohol and cannabis dependence and abuse were subjected to a taxometric analysis using data from a large epidemiological survey of mental disorders in the general population.

Results: Alcohol use problems were best explained by a latent, continuous dimension ranging from mild to severe, while problems associated with using cannabis were best explained by a latent discrete category.

Conclusions: These findings have implications for the way that substance use problems are measured and classified and show specificity with regard to the type of substance under investigation.

\section{Diagnosing mild cognitive impairment: the problem with subjective complaints}

\author{
M Slavin, H Brodaty, N Kochan, P Sachdev
}

The University of New South Wales, Sydney, Australia

Subjective cognitive complaints (SCCs) are common in older adults with up to $88 \%$ of people aged over 85 years complaining of memory problems. The concept of SCCs is ill defined even though they comprise the first of Petersen's diagnostic criteria for mild cognitive impairment (MCI). There is little agreement on how to operationalize SCCs - is it sufficient to simply ask 'have you noticed problems with your memory or thinking?' Nonmemory cognitive complaints have been relatively ignored. The criterion is sensitive but not specific because SCCs are so common. Evidence suggests that SCCs correlate poorly with objective cognitive impairments. It is therefore important to examine the most useful way to define SCCs and whether they contribute significantly to the diagnosis of MCI. We are currently conducting a community study of memory and aging, during which each participant completes three different measures of SCC - two focused on memory and one that covers other cognitive domains. We present data from 300 participants aged $70-90,35 \%$ of whom have cognitive impairment on neuropsychological testing. Cognitive impairment was defined as a result 1.5 SDs or more below normal for age. Cognitive complaints were common in our group - with each measure of SCC being affirmed by up to $66 \%$ of participants. We confirmed a lack of correlation between SCCs and objective cognitive impairment. No one measure of SCC was shown to be more accurate at predicting objective impairment than another. In conclusion, we question whether it is appropriate to preserve $\mathrm{SCC}$ as a diagnostic criterion for MCI.

\section{Collective trauma: the case for a multilevel social-ecological perspective}

\section{Somasundaram \\ University of Adelaide, Adelaide, Australia}

Background: Complex emergencies that follow war and natural disasters have an impact on not only the 